\title{
Patient-level analysis of incident vancomycin-resistant enterococci colonization and antibiotic days of therapy
}

\author{
J. A. McKINNELL ${ }^{1,2,3 *}$, D. F. KUNZ ${ }^{4}$, S. A. MOSER ${ }^{5}$, S. VANGALA ${ }^{6}$, \\ C.-H. TSENG ${ }^{6}$, M. SHAPIRO ${ }^{7}$ AND L. G. MILLER ${ }^{1,2}$ \\ ${ }^{1}$ Infectious Disease Clinical Outcomes Research Unit (ID-CORE), Division of Infectious Disease, Los Angeles \\ Biomedical Research Institute at Harbor-UCLA Medical Center, Torrance, CA, USA \\ ${ }^{2}$ David Geffen School of Medicine at the University of California, Los Angeles, Westwood, CA \\ ${ }^{3}$ Torrance Memorial Medical Center, Torrance, CA, USA \\ ${ }^{4}$ Division of Infectious Disease, University of Alabama at Birmingham, Birmingham, AL, USA \\ ${ }^{5}$ Department of Pathology, University of Alabama at Birmingham, Birmingham, $A L, U S A$ \\ ${ }^{6}$ Department of Medicine Statistics Core, University of California, Los Angeles, Westwood, CA, USA \\ ${ }^{7}$ Division of General Internal Medicine and Health Services Research, University of California, Los Angeles; \\ Los Angeles, CA, USA
}

Received 21 July 2015; Final revision 18 November 2015; Accepted 19 November 2015; first published online 9 December 2015

\section{SUMMARY}

Vancomycin-resistant enterococci (VRE) infections are a public health threat associated with increased patient mortality and healthcare costs. Antibiotic usage, particularly cephalosporins, has been associated with VRE colonization and VRE bloodstream infections (VRE BSI). We examined the relationship between antimicrobial usage and incident VRE colonization at the individual patient level. Prospective, weekly surveillance was undertaken for incident VRE colonization defined by negative admission but positive surveillance swab in a medical intensive care unit over a 17-month period. Antimicrobial exposure was quantified as days of therapy (DOT)/1000 patient-days. Multiple logistic regression was used to analyse incident VRE colonization and antibiotic DOT, controlling for demographic and clinical covariates. Ninety-six percent (1398/1454) of admissions were swabbed within $24 \mathrm{~h}$ of intensive care unit (ICU) arrival and of the 380 patients in the ICU long enough for weekly surveillance, $83(22 \%)$ developed incident VRE colonization. Incident colonization was associated in bivariate analysis with male gender, more previous hospital admissions, longer previous hospital stay, and use of cefepime/ceftazidime, fluconazole, azithromycin, and metronidazole $(P<0 \cdot 05)$. After controlling for demographic and clinical covariates, metronidazole was the only antibiotic independently associated with incident VRE colonization (odds ratio $2 \cdot 0,95 \%$ confidence interval $1 \cdot 2-3 \cdot 3, P<0 \cdot 009$ ). Our findings suggest that risk of incident VRE colonization differs between individual antibiotic agents and support the possibility that antimicrobial stewardship may impact VRE colonization and infection.

Key words: Antibiotics, Enterococcus, Gram-positive bacteria.

\footnotetext{
* Author for correspondence: J. A. McKinnell, MD, Infectious Disease Clinical Outcomes Research Unit, Harbor-UCLA Division of Infectious Disease, David Geffen School of Medicine, 1124 West Carson Street, Box 466, Torrance, CA 90502, USA. (Email: Dr.McKinnell@yahoo.com)
}

\section{INTRODUCTION}

Vancomycin-resistant enterococcal (VRE) infections are the fourth leading cause of mortality from antibiotic-resistant pathogens in the United States [1]. Despite efforts from the Centers for Disease Control 
and Prevention, Infectious Disease Society of America, Society for Hospital Epidemiology of America, and hospital infection control programmes, VRE continues to pose a rising health threat to patients [1-7]. Indeed, Enterococcus spp. are now the second most common cause of central line associated bloodstream infection, and vancomycin resistance is rising [4].

As a complement to infection control measures, antimicrobial stewardship may have the potential to reduce VRE infections and multiple investigations have reported reductions in VRE infections in hospitals that restrict all cephalosporin antibiotics [8-16]. However, antimicrobial stewardship against an entire class of antimicrobials poses significant logistical challenges as a loss of a drug class can limit therapeutic options at a time when antibiotic choices are decreasing. Moreover, there is controversy over which agent might be used to replace cephalosporins $[17,18]$.

While the data associating cephalosporin usage and VRE infection are fairly consistent, the data on individual antibiotics and risk of VRE colonization remain unclear. Hospital-level epidemiological data and animal models suggest that piperacillin/tazobactam is a lower risk than cephalosporin antibiotics for incident VRE colonization [19-23] but a patientlevel analysis of incident VRE colonization from Pittsburgh, USA found no association between this and the use of piperacillin/tazobactam [18]. In fact, a relatively recent study from another US hospital, which included microbiome analysis, specifically implicated metronidazole with incident VRE colonization and not piperacillin/tazobactam or cephalosporin antibiotics [24]. We consider that a better understanding of antibiotic exposure and VRE colonization is important because of the well-established association between colonization pressure and risk of subsequent VRE infections [25]. The aim of this investigation was to analyse patient-level data from an intensive VRE surveillance programme to compare the association between individual antimicrobial agents and incident VRE colonization.

\section{METHODS}

\section{Study design and setting}

A prospective, patient-level surveillance programme for acquisition of VRE rectal colonization during intensive care unit (ICU) stay, i.e. incident VRE colonization in the medical ICU (MICU) was conducted at the University of Alabama at Birmingham Medical Center
(UAB). UAB is an urban tertiary-care hospital with over 900 beds and eight adult ICUs. The MICU is a 33-bed unit of single patient rooms focused on noncardiac, non-surgical intensive care and was chosen for this investigation because it has the highest incidence rate ratio of VRE BSI among ICUs at UAB [26].

\section{Monitoring for VRE colonization}

Monitoring for VRE colonization involved scheduled rectal swabbing and routine culture techniques. Using a pre-specified admission order set, patients had a baseline rectal swab collected on the day of admission to the MICU (baseline screening swab). Every Tuesday, all patients in the unit had a subsequent rectal swab obtained (unit surveillance swab). Perirectal swabbing was allowed to avoid rectal contact in immunocompromised patients [27]. Enhanced VRE surveillance was in place in the MICU starting in April 2009. The investigation utilized data from March 2010 to July 2011, because compliance with the surveillance protocol was $<70 \%$ prior to initiation of the pre-specified admission order set in January 2010. The samples were suspended in $100 \mu \mathrm{l}$ sterile water and plated on an Enterococcosel (BBL) medium containing $6 \mu \mathrm{g}$ vancomycin/ml (Sigma, USA).

The swabbing procedures were conducted by nursing staff as a matter of routine infection control surveillance to allow for initiation of contact isolation. The implemented protocol was used for outcome assignments. The incident-colonized group was defined by a negative screen followed by positive surveillance and the never-colonized group was defined by a negative screen and negative surveillance. Standard infection control policies including contact isolation, the use of dedicated instruments and equipment, and the cleaning and disinfecting of contaminated items for patients with multidrug-resistant organisms and Clostridium difficile were in place during the course of the study.

\section{Antimicrobial utilization}

Antimicrobial usage was evaluated at the patient level. The method utilized was based on individual patient charge data in which a day of therapy was represented by a patient receiving at least one dose of the selected antibiotic. Previous investigations showed that charge data accurately reflected antibiotic usage at UAB [26] and therefore charge data were collected for all antibacterial and antifungal agents used in the study cohort during the study period. Data on antiviral and 
antiretroviral medications were not collected. Medication usage was quantified as days of therapy (DOT)/1000 patient-days. DOT was measured for all antibiotics during the period of hospitalization leading up to final VRE testing. Cefepime and ceftazidime were combined into a single antibiotic class as antipseudomonal cephalosporins. All other antibiotics were analysed independently. All antibiotics with $>50$ DOT in the study cohort were included in the analysis.

Availability of antimicrobial agents on the formulary was dependent on pharmaceutical acquisition as negotiated by the hospital. Antimicrobial stewardship programmes limiting fluoroquinolone use were in place during the entire study period. Specifically, fluoroquinolones could only be prescribed with the oral consent of an infectious disease specialist.

\section{Clinical predictors and statistical analysis}

Demographic data were collected from the medical record on all patients admitted to the MICU, including age, gender, and race/ethnicity. In addition, previous hospital exposure was collected and defined as number of previous hospitalizations in the preceding 365 days, and hospital length of stay prior to transfer to the MICU. Analysis of patient data was restricted to the first admission at which a minimum of two swabs were taken.

Categorical variables were compared between incident VRE and never-colonized VRE patients using Pearson $\chi^{2}$ tests. Antibiotic DOT variables that were highly skewed were compared using Wilcoxon ranksum tests. Other continuous variables were compared using Student's $t$ test. A logistic regression analysis was performed to predict incident VRE colonization (vs. never colonized), with the main exposure variables of antibiotic DOT, while controlling for baseline demographic and clinical covariates. A Cox proportional hazards model of time to incident VRE was also performed for comparison. A $P$ value $<0.05$ was considered statistically significant. All statistical analyses were performed using SAS v. 9.3 (SAS Institute Inc., USA).

\section{RESULTS}

There were 1454 patient admissions to the MICU from 1 March 2010 to 1 July 2011. Compliance with baseline rectal swab within $24 \mathrm{~h}$ of admission was $>96 \%(n=1398 / 1454)$ and screening identified 179
$(13 \%)$ as VRE colonized on admission. The admission surveillance system resulted in an additional 2647 hospital days of contact isolation (1398 tests, 1.9 contact precaution days per test) compared to no surveillance. Patients who were colonized on admission were 53\% male, 58\% Caucasian, had a mean age of 58 years $($ s.D. $=16)$, and were in the hospital prior to ICU admission on average for 19 days (S.D. $=32$ ).

Of the 1219 patients who were not colonized on admission, $380(31 \%)$ remained in the ICU long enough to undergo weekly VRE unit surveillance which identified $83(22 \%)$ patients who developed incident VRE colonization and $297(78 \%)$ patients that never became colonized. The weekly surveillance system resulted in an additional 1549 hospital days of contact isolation (527 tests, 2.9 contact precaution days per test; see Figs 1 and 2). Compared to the 297 patients who never became colonized, the 83 patients who developed incident VRE colonization were more frequently male $(60 \%$ vs. $47 \%, P=0.03)$, had more previous admissions $(2 \cdot 2 \pm 3 \cdot 3$ vs $1 \cdot 6 \pm 1 \cdot 2$ admissions, $P=$ $0 \cdot 01)$ and a longer hospital length of stay prior to ICU admission (6.6 \pm 13.9 vs. $1.8 \pm 4.2$ hospital days, $P<0 \cdot 0001)$.

Antibiotics were commonly prescribed to the 380 patients who remained in the ICU long enough to undergo weekly VRE unit surveillance. Only nine antimicrobials were used for $>50$ DOT/1000 patient-days (Table 1) Antibiotic exposure differed between incidentcolonized and never-colonized patients. Cefepime/ceftazidime DOT/1000 patient-days was higher in incident VRE-colonized patients $(P=0 \cdot 02)$. Azithromycin $(P=$ $0 \cdot 007)$, fluconazole $(P=0.02)$ and metronidazole $(P=$ $0 \cdot 001) \mathrm{DOT} / 1000$ patient-days were also higher in incident VRE-colonized patients. There was a nonsignificant trend toward higher piperacilin/tazobactam in the never-colonized cohort $(P=0 \cdot 16)$.

The multivariable logistic regression analysis identified patient age and hospital length of stay prior to ICU admission as significant predictors of incident VRE colonization. Of the antibiotics, only metronidazole was independently associated with incident VRE colonization (odds ratio $2 \cdot 0,95 \%$ CI 1·2-3·3, $P=0 \cdot 009$ ) (Table 2). The Cox proportional hazards model yielded very similar results to the logistic regression model.

\section{DISCUSSION}

In our analysis of VRE colonization and antibiotic usage on 1454 admissions to a tertiary-care MICU, including 83 cases of incident VRE colonization, we 


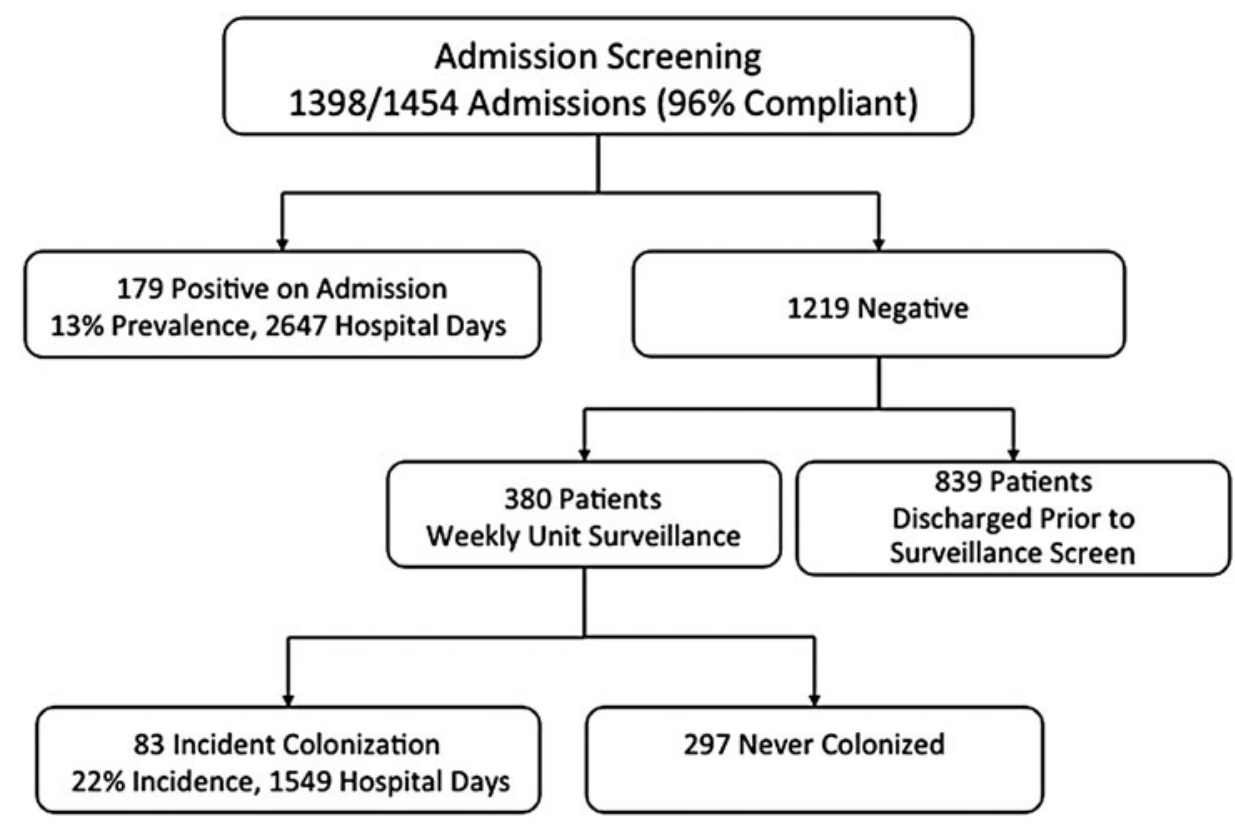

Fig. 1. Flow diagram of patients through the study, including yield of vancomycin-resistant enterococci (VRE) admission testing $v s$. weekly VRE surveillance.

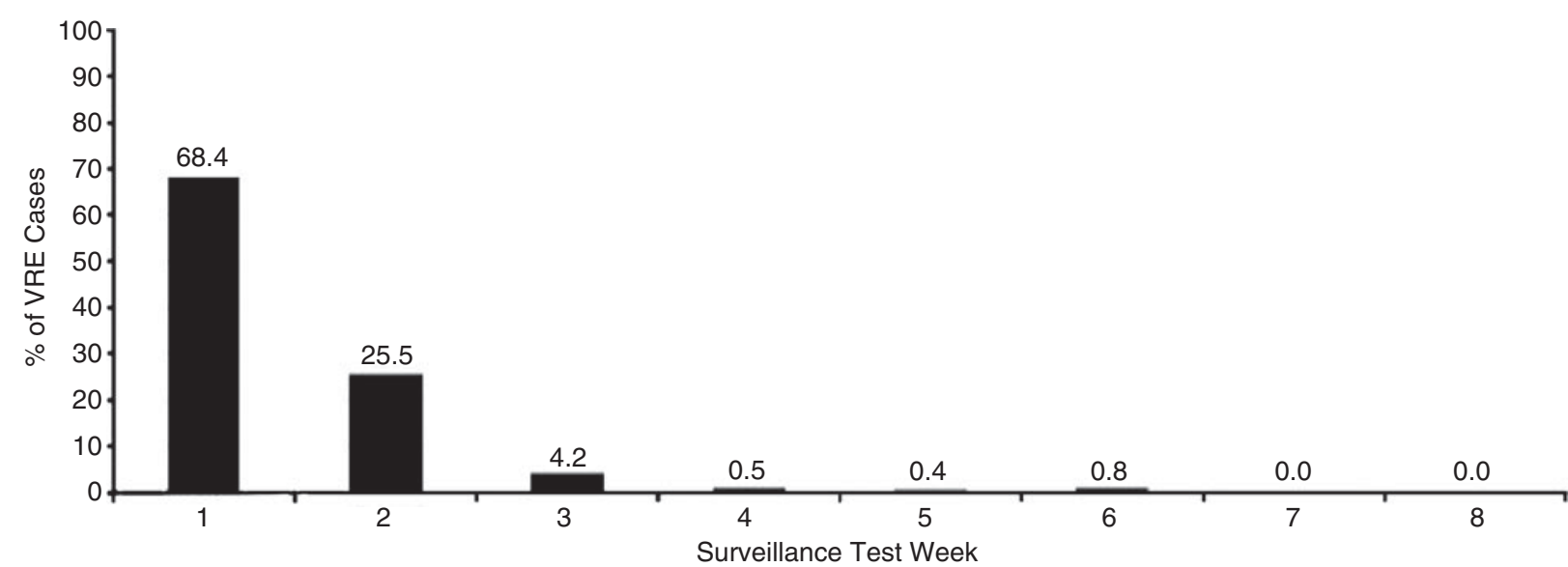

Fig. 2. Proportion of vancomycin-resistant enterococci cases detected at admission (test no. 1) and through weekly surveillance (test no. 2) in medical intensive care unit.

noted differences in antibiotic exposure between patients who developed VRE colonization and patients who were never colonized with VRE. Our investigation includes 17 months of surveillance data collected from a high VRE burden environment.

One of our primary a priori objectives for this study was to compare individual cephalosporins against pipercillin/tazobactom in terms of their association with risk of incident VRE colonization. In bivariate analysis, an association was evident between cefepime/ceftazidime DOT and VRE colonization as well as a weak trend toward a protective effect of pipercillin-tazobactam $(P=0 \cdot 16)$. However, the association between cefepime/ceftazidime and incident VRE colonization did not persist in multivariable analysis. These data suggest that restriction of later generation cephalosporins may not result in significant reductions in VRE colonization risk [18]. However, caution should be exercised when interpreting our data. Published reports from multiple hospitals have documented significant reductions in VRE incidence following complete restriction of all cephalosporin antibiotics [8-16]. Moreover, previous epidemiological data from UAB has shown that ceftriaxone is more 
Table 1. Simple logistic comparison of patients' characteristics and antibiotic DOT between incident VRE-colonized and never VRE-colonized patients $(n=379)$.

\begin{tabular}{|c|c|c|c|}
\hline & $\begin{array}{l}\text { Incident } \\
\text { VRE }(N=83)\end{array}$ & $\begin{array}{l}\text { Never colonized } \\
(N=297)\end{array}$ & $P$ value \\
\hline Age, yr & & & $0 \cdot 055$ \\
\hline Mean (S.D.) & $61(15 \cdot 8)$ & $57(15 \cdot 7)$ & \\
\hline Sex & & & 0.03 \\
\hline Male, $n(\%)$ & $50(60 \cdot 2)$ & $139(46 \cdot 8)$ & \\
\hline Race/ethnicity & $0 \cdot 48$ & & \\
\hline White, $n(\%)$ & $44(55 \cdot 7)$ & $173(60 \cdot 1)$ & \\
\hline Non-white, $n(\%)$ & $35(44 \cdot 3)$ & $115(39 \cdot 9)$ & \\
\hline Previous admissions in last 365 days & & & 0.01 \\
\hline Mean (S.D.) & $2 \cdot 2(3 \cdot 3)$ & $1 \cdot 6(1 \cdot 2)$ & \\
\hline Days in wards prior to ICU admission & & & $<0.0001$ \\
\hline Mean (S.D.) & $6 \cdot 6(13 \cdot 9)$ & $1 \cdot 8(4 \cdot 2)$ & \\
\hline Azithromycin, DOT/1000 pd & & & 0.007 \\
\hline Mean (s.D.) & $228(357 \cdot 0)$ & $133(267 \cdot 4)$ & \\
\hline Median (Q1-Q3) & $0(0-400)$ & $0(0-100)$ & \\
\hline Cefepime/ceftazidime, DOT/1000 pd & & & $\mathbf{0 . 0 2}$ \\
\hline Mean (s.D.) & $239(353 \cdot 6)$ & $161(288 \cdot 3)$ & \\
\hline Median (Q1-Q3) & $0(0-400)$ & $0(0-250)$ & \\
\hline Ceftriaxone, DOT/1000 pd & & & 0.56 \\
\hline Mean (s.D.) & $61(152 \cdot 8)$ & $119(278 \cdot 3)$ & \\
\hline Median (Q1-Q3) & $0(0-0)$ & $0(0-0)$ & \\
\hline Fluconazole, DOT/1000 pd & & & $0 \cdot 0157$ \\
\hline Mean (S.D.) & $77(183 \cdot 0)$ & $41(132 \cdot 0)$ & \\
\hline Median (Q1-Q3) & $0(0-0)$ & $0(0-0)$ & \\
\hline Metronidazole, DOT/1000 pd & & & $0 \cdot 0013$ \\
\hline Mean (s.D.) & $171(290 \cdot 8)$ & $89(222 \cdot 4)$ & \\
\hline Median (Q1-Q3) & $0(0-250)$ & $0(0-0)$ & \\
\hline Moxifloxacin, DOT/1000 pd & & & $0 \cdot 7990$ \\
\hline Mean (S.D.) & $68(185 \cdot 0)$ & $82(226 \cdot 4)$ & \\
\hline Median (Q1-Q3) & $0(0-0)$ & $0(0-0)$ & \\
\hline Piperacillin/tazobactam, DOT/1000 pd & & & $0 \cdot 16$ \\
\hline Mean (S.D.) & $433(380 \cdot 3)$ & $528(456 \cdot 8)$ & \\
\hline Median (Q1-Q3) & $381 \cdot 0(35 \cdot 7-769 \cdot 2)$ & $500(0-1000)$ & \\
\hline Tobramycin, DOT/1000 pd & & & $0 \cdot 4630$ \\
\hline Mean (s.D.) & $58(141 \cdot 6)$ & $71(193 \cdot 5)$ & \\
\hline Median (Q1-Q3) & $0(0-33)$ & $0(0-0)$ & \\
\hline Vancomycin, DOT (IV formulation)/1000 pd & & & $0 \cdot 3191$ \\
\hline Mean (S.D.) & $490(313 \cdot 3)$ & $541(384 \cdot 9)$ & \\
\hline Median (Q1-Q3) & $433(294-750)$ & $500(222-800)$ & \\
\hline
\end{tabular}

VRE, Vancomycin-resistant enterococci; DOT/1000 pd, days of therapy per 1000 person-days.

closely associated with VRE colonization than other cephalosporin antibiotics [26]. Our multivariate analysis did not find an association between ceftriaxone use and incident VRE colonization, but it is noteworthy that the use of ceftriaxone in this study cohort was low, approximately one-quarter of that of cefepime/ceftazidime and $<15 \%$ of that of piperacillin/ tazobactam, therefore the study may have been underpowered to detect such a relationship.

Strong bivariate associations were evident between azithromycin, fluconazole, and metronidazole DOT and VRE colonization, suggesting that the relationship between host intestinal microflora, emergence of VRE, and antibiotic exposure may be relatively dynamic and complex [24]. Of the antimicrobial agents used in our cohort, metronidazole was independently associated with increased risk of incident VRE colonization. Such an association has been previously shown in a mouse model of VRE persistence [21], but not for VRE acquisition [28]. In human studies, anti-anaerobic agents have been consistently associated with higher density of VRE colonization [15, 
Table 2. Multiple logistic regression model of incident VRE colonization in terms of antibiotic DOT, with clinical predictor variables

\begin{tabular}{lll}
\hline \hline Effect & OR $(95 \% \mathrm{CI})$ & $P$ value \\
\hline Cefepime/ceftazidime, DOT & $1 \cdot 2(0 \cdot 8-1 \cdot 9)$ & $0 \cdot 45$ \\
Ceftriaxone, DOT & $0 \cdot 5(0 \cdot 3-1 \cdot 2)$ & $0 \cdot 13$ \\
Piperacillin/tazobactam, DOT & $0 \cdot 84(0 \cdot 6-1 \cdot 2)$ & $0 \cdot 39$ \\
Azithromycin & $1 \cdot 2(0 \cdot 8-1 \cdot 8)$ & $0 \cdot 48$ \\
Fluconazole & $1 \cdot 4(0 \cdot 6-3 \cdot 3)$ & $0 \cdot 44$ \\
Metronidazole & $\mathbf{2 \cdot 0}(\mathbf{1 \cdot 2}-\mathbf{3} \cdot \mathbf{3})$ & $\mathbf{0 \cdot 0 0 9}$ \\
Previous admissions & $1 \cdot 2(0 \cdot 99-1 \cdot 3)$ & $0 \cdot 06$ \\
Days in hospital prior to ICU & $1 \cdot 8(1 \cdot 3-2 \cdot 4)$ & $\mathbf{0} \cdot \mathbf{0 0 0 3}$ \\
$\quad(>1$ week) & $1 \cdot 2(1 \cdot 0-1 \cdot 5)$ & $\mathbf{0 \cdot 0 2}$ \\
Age (>10 years) & $1 \cdot 8(1 \cdot 0-3 \cdot 16)$ & $0 \cdot 04$ \\
Sex: Male & $1 \cdot 3(0 \cdot 77-2 \cdot 4)$ & $0 \cdot 30$ \\
Race: Non-white & \\
\hline \hline
\end{tabular}

VRE, Vancomycin-resistant enterococci; DOT, days of therapy; OR, odds ratio; CI, confidence interval.

19, 29]. Notably, in a detailed analysis of 94 patients undergoing allogenic haematopoietic stem cell transplantation, Taur and colleagues demonstrated that enterococcal overgrowth was increased threefold by metronidazole administration [24]. Unfortunately, our investigation, like the latter study, did not capture the reason for metronidazole administration. Therefore, some of the association seen with metronidazole may be influenced by clinical antibiotic selection bias or differences in underlying comorbid conditions. Nevertheless, a relatively consistent picture emerges to associate anti-anaerobic therapy, particularly metronidazole with VRE colonization.

Last, our data indicate that older age and longer hospitalization were independent predictors of incident VRE colonization. Recent animal model studies have highlighted the role of gastrointestinal mucosal immunity in preventing VRE colonization [30]. Further, increasing age has long been associated with immune senescence within the gastrointestinal mucosa, best described in cases of $C$. difficile infections that may provide biological plausibility for the observed association of age and VRE colonization [31, 32]. Our findings support the notion that sicker and more vulnerable patients are at highest risk for acquiring VRE and represent an appropriate population for antimicrobial stewardship initiatives or other infection control efforts. There may be an opportunity to explore human host immunological function, particularly at the gastrointestinal mucosa level, as it relates to risk of VRE colonization.
The association between length of hospitalization prior to ICU admission and incident VRE colonization was unexpected and warrants further investigation. One possibility is that hospitalization results in gastrointestinal dysbiosis that predisposes to emergence or acquisition of VRE during ICU stay. Future investigations should include serial microbiome analysis to further explore this hypothesis.

Our study has some important limitations. Perhaps most significantly, we did not conduct surveillance of patients on discharge from the ICU. Due to the logistics of patient sampling, patients were only swabbed on Tuesdays and so the outcomes are unknown for $70 \%$ of patients who were discharged from the unit prior to weekly surveillance. Further, the weekly surveillance strategy may have resulted in both selection and misclassification bias and likely underestimates incident VRE colonization. In addition, our surveillance of VRE resulted in a binary outcome and a quantitative approach such as VRE density in stool may have provided better sensitivity to detect differences between individual antibiotics. The study did not influence or control antibiotic prescribing and we were also unable to control for reasons for antibiotic prescribing. Last, as mentioned earlier the study was underpowered to examine the relationship between VRE colonization and subsequent VRE infection.

Future research into VRE prevention is needed as its incidence is increasing in many US hospitals, particularly tertiary medical centres that care for highly immunocompromised and vulnerable patients [33]. There is potential for the use of antibiotic stewardship programmes as an adjunct to infection control interventions (e.g. improved hand hygiene, barrier precautions) at preventing VRE colonization [2]. Unfortunately, previous published reports have depended on absolute restriction of all cephalosporins which may not be practical in some settings. Ultimately, success of antimicrobial stewardship programmes will depend on developing a feasible approach that is amenable to widespread dissemination. Data from our investigation also highlight the need to better understand the relationship between individual antibiotics and the host's intestinal flora, including normal commensal organisms, VRE, and other healthcare pathogens in order to inform providers and antimicrobial stewardship programmes on best practices for responsible antibiotic prescribing.

\section{ACKNOWLEDGEMENTS}

J.M. received support from the NIH/NCRR/NCATS UCLA CTSI grant no. KL2TR 000 122. C.T. received 
support from the University of California, Los Angeles, Resource Centers for Minority Aging Research Center for Health Improvement of Minority Elderly (RCMAR/CHIME) under NIH/ NIA grant no. P30-AG 021684 , and the UCLA CTSI under NIH/NCRR/NCATS grant no. UL1TR 000 124. S.V. received support from the UCLA CTSI under NIH/NCRR/NCATS grant no. UL1TR 000124 . The content does not necessarily represent the official views of the NIA or the NIH.

\section{DECLARATION OF INTEREST}

None.

\section{REFERENCES}

1. Centers for Disease Control and Prevention. Vital signs: carbapenem-resistant Enterobacteriaceae. Morbidity and Mortality Weekly Report 2013; 62: 165-170.

2. Siegel JD, et al. Management of multidrug-resistant organisms in health care settings, 2006. American Journal of Infection Control 2007; 35 (Suppl. 2): S165193.

3. Boucher HW, et al. Bad bugs, no drugs: no ESKAPE! An update from the Infectious Diseases Society of America. Clinical Infectious Diseases 2009; 48: 1-12.

4. Hidron AI, et al. NHSN annual update: antimicrobialresistant pathogens associated with healthcareassociated infections: annual summary of data reported to the National Healthcare Safety Network at the Centers for Disease Control and Prevention, 2006-2007. Infection Control and Hospital Epidemiology 2008; 29: 996-1011.

5. Wisplinghoff $\mathbf{H}$, et al. Nosocomial bloodstream infections in US hospitals: analysis of 24,179 cases from a prospective nationwide surveillance study. Clinical Infectious Diseases 2004; 39: 309-317.

6. Deshpande LM, et al. Antimicrobial resistance and molecular epidemiology of vancomycin-resistant enterococci from North America and Europe: a report from the SENTRY antimicrobial surveillance program. Diagnostic Microbiology and Infectious Diseases 2007; 58: $163-170$.

7. System NNIS. National Nosocomial Infections Surveillance (NNIS) System Report, data summary from January 1992 through June 2004, issued October 2004. American Journal of Infection Control 2004; 32: 470-485.

8. Quale J, et al. Manipulation of a hospital antimicrobial formulary to control an outbreak of vancomycinresistant enterococci. Clinical Infectious Diseases 1996; 23: $1020-1025$.

9. Bradley SJ, et al. The control of hyperendemic glycopeptide-resistant Enterococcus spp. on a haematology unit by changing antibiotic usage. Journal of Antimicrobial Chemotherapy 1999; 43: 261-266.

10. Montecalvo MA, et al. Infection-control measures reduce transmission of vancomycin-resistant enterococci in an endemic setting. Annals of Internal Medicine 1999; 131: 269-272.

11. Smith DW. Decreased antimicrobial resistance after changes in antibiotic use. Pharmacotherapy 1999; 19: 129S-132S; discussion 33S-37S.

12. Manzella J, et al. Choice of antibiotic and risk of colonization with vancomycin-resistant Enterococcus among patients admitted for treatment of community-acquired pneumonia. Infection Control and Hospital Epidemiology 2000; 21: 789-791.

13. May AK, et al. Reduction of vancomycin-resistant enterococcal infections by limitation of broad-spectrum cephalosporin use in a trauma and burn intensive care unit. Shock 2000; 14: 259-264.

14. Nourse C, et al. Eradication of vancomycin resistant Enterococcus faecium from a paediatric oncology unit and prevalence of colonization in hospitalized and community-based children. Epidemiology and Infection 2000; 124: 53-59.

15. Puzniak LA, et al. Acquisition of vancomycin-resistant enterococci during scheduled antimicrobial rotation in an intensive care unit. Clinical Infectious Diseases 2001; 33: 151-157.

16. Lautenbach E, et al. Changes in the prevalence of vancomycin-resistant enterococci in response to antimicrobial formulary interventions: impact of progressive restrictions on use of vancomycin and third-generation cephalosporins. Clinical Infectious Diseases 2003; 36: 440-446.

17. Stiefel $\mathbf{U}$, et al. Effect of the increasing use of piperacillin/tazobactam on the incidence of vancomycin-resistant enterococci in four academic medical centers. Infection Control and Hospital Epidemiology 2004; 25: 380-383.

18. Paterson DL, et al. Acquisition of rectal colonization by vancomycin-resistant Enterococcus among intensive care unit patients treated with piperacillin-tazobactam versus those receiving cefepime-containing antibiotic regimens. Antimicrobial Agents and Chemotherapy 2008; 52: 465-469.

19. Donskey CJ, et al. Effect of antibiotic therapy on the density of vancomycin-resistant enterococci in the stool of colonized patients. New England Journal of Medicine 2000; 343: 1925-1932.

20. Donskey CJ, et al. Recurrence of vancomycin-resistant Enterococcus stool colonization during antibiotic therapy. Infection Ccontrol and Hospital Epidemiology 2002; 23: 436-440.

21. Donskey CJ, et al. Effect of parenteral antibiotic administration on persistence of vancomycin-resistant Enterococcus faecium in the mouse gastrointestinal tract. Journal of Infectious Diseases 1999; 180: 384-390.

22. Lakticova $\mathbf{V}$, et al. Antibiotic-induced enterococcal expansion in the mouse intestine occurs throughout the small bowel and correlates poorly with suppression of competing flora. Antimicrobial Agents and Chemotherapy 2006; 50: 3117-3123.

23. Rice LB, et al. Beta-lactam antibiotics and gastrointestinal colonization with vancomycin-resistant enterococci. Journal of Infectious Diseases 2004; 189: 1113-1118. 
24. Taur Y, et al. Intestinal domination and the risk of bacteremia in patients undergoing allogeneic hematopoietic stem cell transplantation. Clinical Infectious Diseases 2012; 55: 905-14.

25. Bonten MJ, et al. The role of 'colonization pressure' in the spread of vancomycin-resistant enterococci: an important infection control variable. Archives of Internal Medicine 1998; 158: 1127-1132.

26. McKinnell JA, et al. Association between vancomycinresistant enterococci bacteremia and ceftriaxone usage. Infection Control and Hospital Epidemiology 2012; 33: 718-724.

27. Weinstein JW, et al. Comparison of rectal and perirectal swabs for detection of colonization with vancomycinresistant enterococci. Journal of Clinical Microbiology 1996; 34: 210-212.

28. Donskey CJ, et al. Effect of parenteral antibiotic administration on the establishment of colonization with vancomycin-resistant Enterococcus faecium in the mouse gastrointestinal tract. Journal of Infectious Diseases 2000; 181: 1830-1833.
29. Bhalla A, et al. Antianaerobic antibiotic therapy promotes overgrowth of antibiotic-resistant, gram-negative bacilli and vancomycin-resistant enterococci in the stool of colonized patients. Infection Control and Hospital Epidemiology 2003; 24: 644-649.

30. Kinnebrew MA, et al. Bacterial flagellin stimulates Toll-like receptor 5-dependent defense against vancomycin-resistant Enterococcus infection. Journal of Infectious Diseases 2010; 201: 534-543.

31. Poutanen SM, Simor AE. Clostridium difficileassociated diarrhea in adults. Canadian Medical Association Journal 2004; 171: 51-58.

32. Kyne L, et al. Association between antibody response to toxin A and protection against recurrent Clostridium difficile diarrhoea. Lancet 2001; 357: 189-193.

33. California Department of Public Health. Methicillinresistant Staphylococcus aureus (MRSA) and vancomycin-resistant enterococci (VRE) bloodstream infections (BSI) in California hospitals, 2011 (http://www.cdph.ca. gov/programs/hai/Pages/MRSAandVRE-Report.aspx). 